\title{
A Study on The Capacity of Massive MIMO System with The Aid of MATLAB Simulink
}

\author{
Mohammed H. M. Sadig, and Amin B. A. Mustafa
}

\begin{abstract}
Today's main focus of the emerging cellular technologies is to fulfill users high demand while fully utilizing available resources. Massive MIMO is expected to play a vital role in this development, such as $\mathbf{5 G}$, as it results in noticeably better performance, against the cost of needing more hardware components. This work study the effect of increasing the number of antennas on the system data-rate (capacity). We derive this relationship mathematically for both Single Input Single Output (SISO) and MIMO systems. Further, different from other papers in literature, using MATLAB Simulink we verify this proportionality for three modulation schemes (QPSK, 16 QAM and 64 QAM) and for several MIMO (2x2 and $4 \times 4)$ and Massive MIMO (8x8, 16x16 and 24x24) systems. We finally establish a comparison between that theoretical results and results obtained from MATLAB Simulink.
\end{abstract}

Index Terms-5G; SISO; Massive MIMO; Capacity; MATLAB; Simulink.

\section{INTRODUCTION}

Massive MIMO is planned to be a core component in the design of the new upcoming $5 \mathrm{G}$ technology, since it provides vital and crucial enhancement on different performance metrics (especially system capacity). Apparently, the trade-offs are the added design complexity and of course the need of extra hardware (in terms of transmit and/or receive antennas). However, theses tradeoffs can be overlooked since massive MIMO provides substantial system improvement and satisfies users' high (continuously increasing) demand.

Extensive research is being conducted on the massive MIMO field and on how the increase of the number of antennas can affect system capacity. For instance, authors in [1], analyze the aggregate impact of different hardware impairments on systems with large antenna arrays (Massive

MIMO). They model the residual hardware impairments at the transmitter and receiver as additive distortion noise. However, they present a direct relationship between the number of antenna arrays and the spectral efficiency (System Capacity). Effectively, with different assumptions throughout their work, they show that the capacity with ideal hardware grows without bounds as the number of antenna increases, while under transceiver hardware impairments the lower and upper bounds converge to finite limits, but still the relationship is in direct proportionality. Motivated by the previous works studying the asymptotic analysis of capacity in Massive MIMO systems [2]-[5] the

Published on May 28, 2018

Mohammed H. Sadig is a lecturer at Bayan College for Science \& Technology, Alslama College, and Cambridge International College, Sudan. (e-mail: mhyder09@live.com)

Dr. Amin B. Mustafa is with the Electrical Engineering Department, Alneelain University (amin31766@gmail.com) authors in [6] provide a nonasymptotic analysis of the capacity for a point-to-point massive MIMO system. They further develop not only deterministic bounds on the ergodic capacity but also statistical bounds on the instantaneous capacity. Ultimately, for both deterministic bounds on ergodic capacity and statistical bounds on instantaneous capacity, they show that as the number of transmit antennas increases, the bounds on both ergodic and instantaneous capacities increase accordingly. Recent work in [7] study the capacity of massive MIMO-OFDM systems with strong nonlinear distortion effects. They derive theoretical expressions for the channel capacity considering different downlink scenarios where a base-station with nonlinear transmitting branches communicate with receive antennas. It is shown that, at the same SNR value the capacity increases as $\mathrm{T}$ increases (the number of transmit antennas). Furthermore, they show that although nonlinear distortion effects can reduce the system capacity, this capacity loss can be reduced by increasing the number of transmit antennas as well.

In this Paper, we first derive a mathematical model that relates the number of antennas to the capacity of the system. Section II effectively builds the argument starting from a SISO case where only single antenna transmitter and single antenna receiver are considered, then we develop an equation for MIMO in general that can be applied for any transmission system with a number of antennas greater than one. Moreover, in section III, as a novel work different from the papers [1], [6], [7], we provide the system model using building blocks of MATLAB Simulink, we discuss only one specific model that can be easily generalized for any less number of antennas. Finally, in Section IV, we show the results from the simulation for capacity vs number of antennas for different modulation schemes and different MIMO settings at the same SNR.

\section{THEORETICAL APPROACH}

In this section we provide an abstract mathematical reasoning in order to be able to conclude that there is a direct impact of the increase of the number of antennas on the system capacity. Therefore, as a start, Single Input Single Output (SISO) system is considered. Claude Shannon showed that the maximum error-free bit for an Additive White Gaussian Noise (AWGN) channel for such a SISO system is given by:

$$
C=B \times \log _{2}\left(1+\frac{S}{N_{0}}\right)
$$

where $\mathrm{C}$ is the channel capacity $(\mathrm{b} / \mathrm{s}), \mathrm{B}$ is the channel bandwidth $(\mathrm{Hz})$ and $\mathrm{S} / \mathrm{N} 0$ is the signal-to-noise power ratio (watts/watts) at the input to the digital receiver. The system 
capacity is defined as the Shannon capacity adjusted by the inherent system losses. From the equation 1, considering fixed channel bandwidth, It is obvious that this capacity is a function of only the SNR.

On the other hand, MIMO is used to increase the signal-to noise ratio. In a system with $\mathrm{T}$ transmit antennas and $\mathrm{R}$ receive antennas, the received SNR can increase in proportion to $\mathrm{T} x \mathrm{R}$. In general, for small $\mathrm{x}$, we can use the approximation $\log 2(1+\mathrm{x})=\mathrm{x}$ for equation 1 . It implies that at lower SNR the capacity increases proportional to the SNR. In the same way, for higher $x$ we have the approximation $\log 2(1+x)=\log 2(x)$. It means at higher SNR the capacity increases logarithmically with the SNR. Thus in the case of multiple antennas at the transmitter and the receiver, there is a way to have up to $\mathrm{N}=\min [\mathrm{T}, \mathrm{R}]$ parallel channels with $\mathrm{N}$ times lower SNR since the signal power is divided among the channels. In this way the channel capacity becomes

$$
C=B \times N \times \log _{2}\left(1+\frac{R}{T} \cdot \frac{S}{N_{0}}\right)
$$

For different SNR values, we apply (1) and (2) to calculate the capacity for SISO and Different MIMO systems respectively. It can be easily shown from Fig. 1 that the theoretically capacity increases with SNR and more interestingly, capacity at the same SNR increases with the increase of the number of transmit and/or receive antennas.

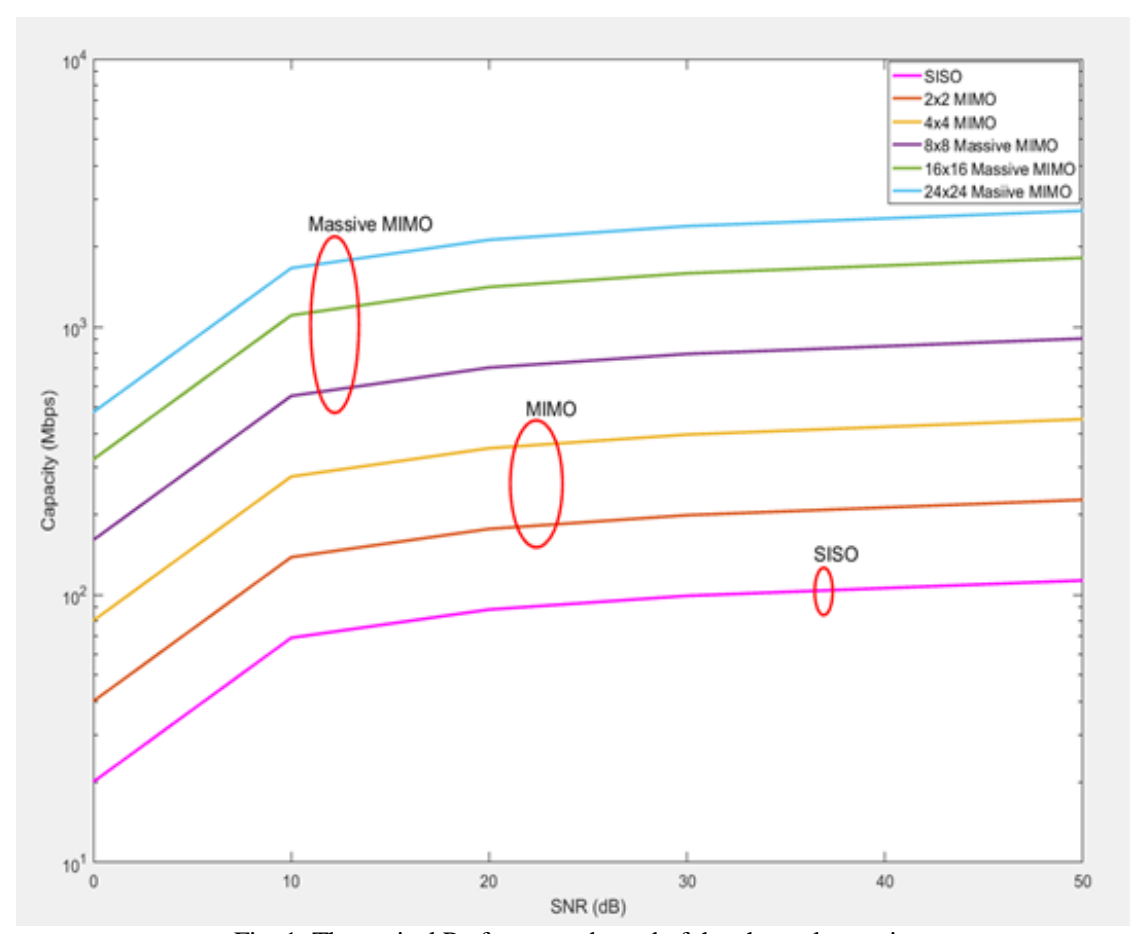

Fig. 1. Theoretical Performance bound of the channel capacity

\section{SySTEM MODEL AND DESIGN EXAMPLE}

In order to prove the theory of the relationship between the capacity and the number of antennas, in this section we implement different MIMO settings using Matlab Simulink building blocks. Namely, we have designed MMO (2x2), $(4 \times 4),(8 \times 8),(16 \times 16)$ and $(24 \times 24)$ systems each with QPSK, 16QAM and 64QAM modulation scheme. For the sake of shortness, we present only massive MIMO (24x24) system, as it is the most sophisticated system among all.

Twenty-four antennas are used for both transmitter and receiver. OFDM is used alongside with an AWGN Rayleigh faded channel as shown in the Fig. 2. The Bernoulli Binary Generator block generates random binary numbers using a Bernoulli distribution. Modulation and Demodulation blocks are used to change between modulation schemes (QPSK, 16-QAM or 64-QAM). OFDM Transmitter composes of
Selector (Selects or reorders specified elements of a multi dimensional input signal), IFFT (Outputs the inverse fast Fourier transform (IFFT) of a real or complex input by computing radix-2 decimation-in-time (DIT) decimation-infrequency (DIF)) and Reshape (Changes the dimensions of a vector or matrix input signal). The MIMO (24x24) fading channel that composes of input port, twenty-four Selectors, ninety-six Rayleigh Fading blocks, twenty-four Sum blocks, twenty-four OFDM Transmitter blocks, and twenty-four Frame Conversion blocks. The OSTBC Combiner job is to Combine the received signal and channel estimate inputs in accordance with the structure of the orthogonal space-time block code (OSTBC). The OSTBC can be rate 1 for 2 transmit antennas, rate $1 / 2$ or $3 / 4$ for 3 and 4 transmit antennas. Finally, OFDM receiver inverses the job of the OFDM transmitter. 


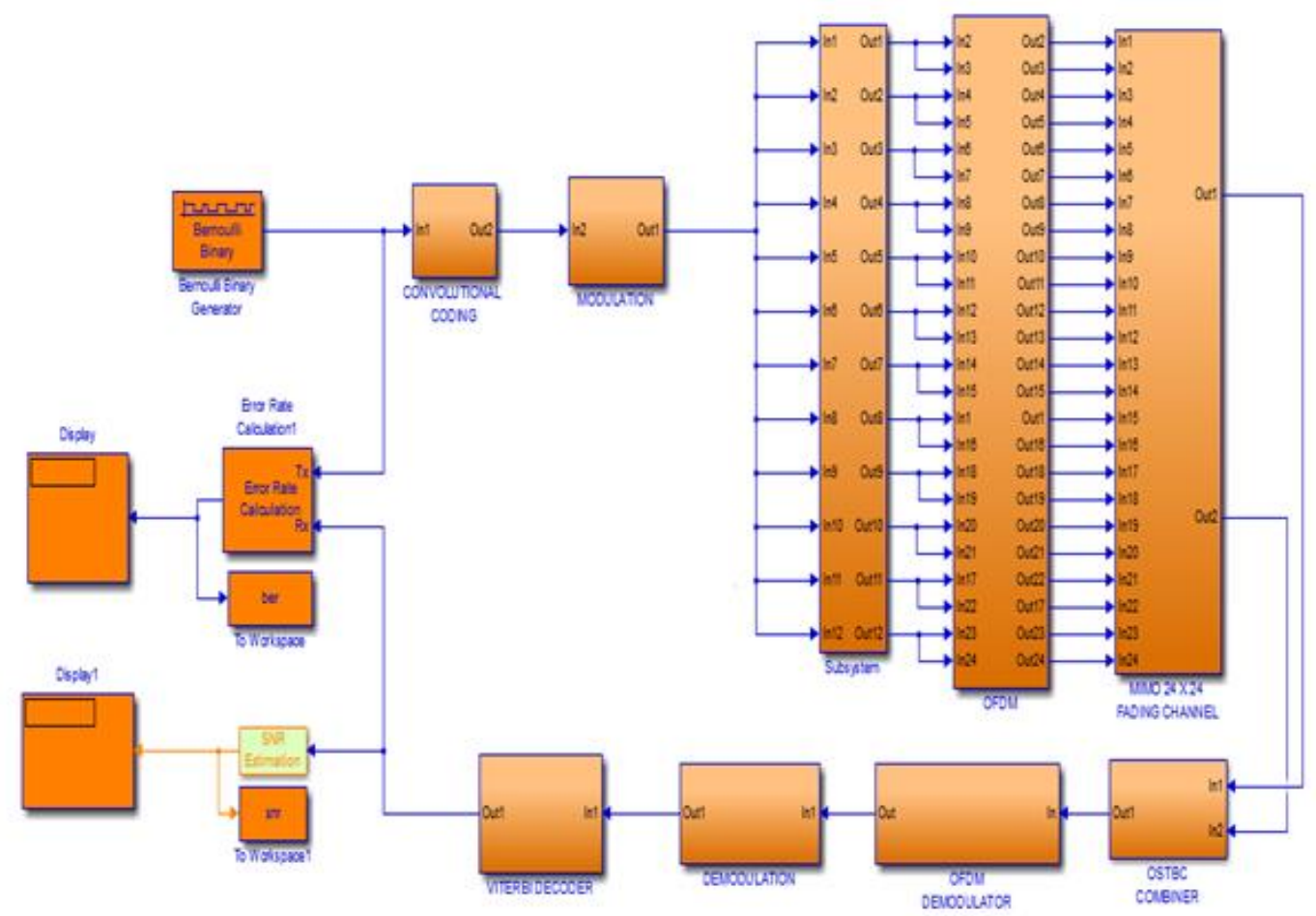

Fig. 2. Massive MIMO (24x24) System Using QAM, 16-QAM,64-QAM Modulation

\section{Simulation Results}

This section provides capacity results for the different MIMO (2x2 and 4x4) and Massive MIMO (8x8, 16x16 and $24 \times 24)$ systems. Results are generated using the MATLAB Simulink blocks described for each system in section III. For MIMO $(2 \times 2)$ with a duration of the simulation set to $100 \mathrm{sec}$, bandwidth of $20 \mathrm{MHz}$. Using QPSK, 16-QAM, 64QAM modulation and OFDM a capacity of (5Mbps-
45Mbps), (20Mbps-45Mbps) and (20Mbps-45Mbps) has been recorded respectively. Same capacities were obtained when MIMO $(4 \times 4)$ is used with code rate.

Furthermore, With the same simulation duration and bandwidth for MIMO $(2 \times 2)$ and $(4 \times 4)$, the capacity is obtained for different massive MIMO systems (8x8), (16x16) and $(24 \times 24)$ using different modulation schemes (QPSK, 16-QAM and 64-QAM). The results for the average capacity of each of the settings can be shown in Fig. 3.

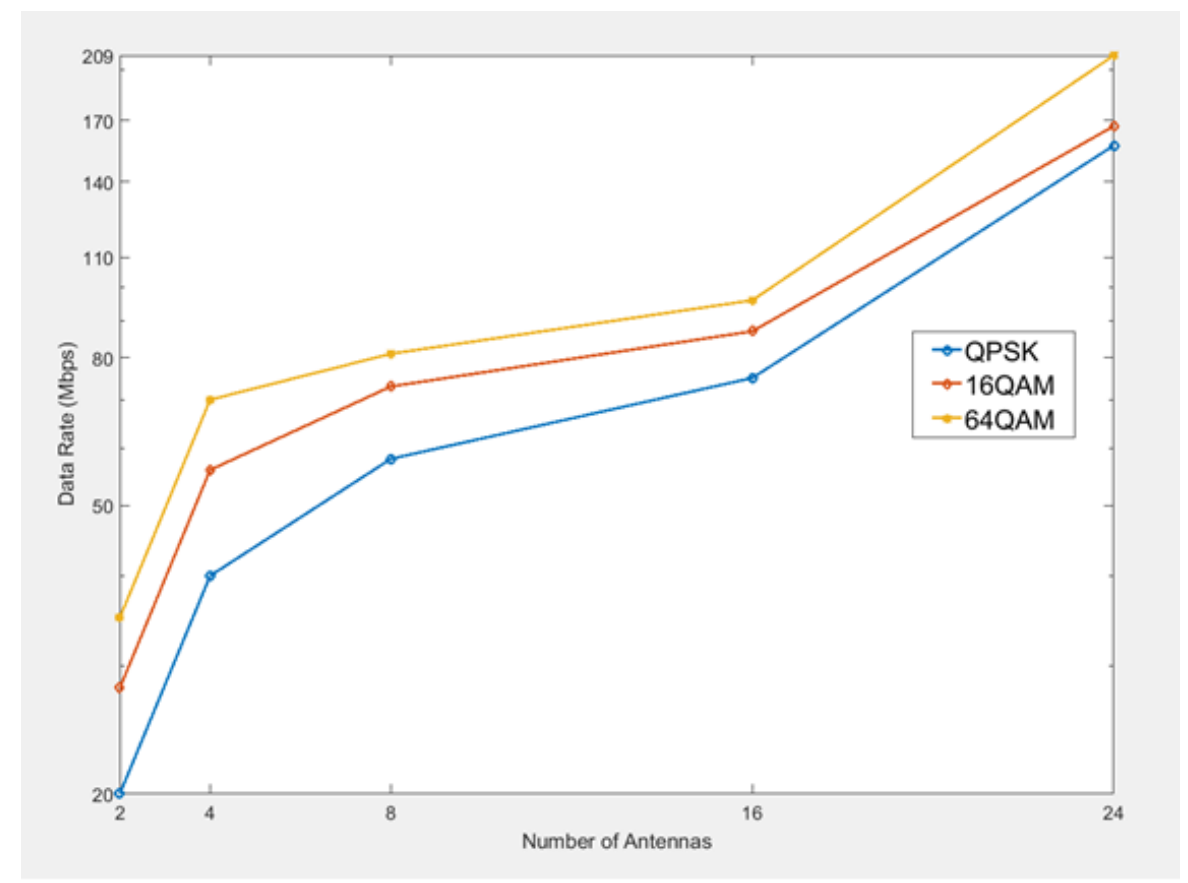

Fig .3. Capacity Vs Number of Antennas for Different Modulation Schemes 


\section{CONCLUSION}

In this paper we studied the impact of increasing the number of both transmission and reception antennas on the capacity of the system. The study has been conducted first from a theoretical approach and it has been shown that for different SNR values ranging from 0 to $50 \mathrm{~dB}$, as the number of antenna increases the capacity linearly increases accordingly. Furthermore, we verified this theoretical outcome using Matlab Simulink to simulate a real system with additive noise and fading gains and it has been shown that also any increase in the number of antennas will result in a capacity increase, this verification has been done for only one SNR value but for different modulation schemes. The conclusion proven to be valid regardless of the modulation scheme.

\section{REFERENCES}

[1] E. Bj"ornson, J. Hoydis, M. Kountouris, and M. Debbah, "Massive mimo systems with non-ideal hardware: Energy efficiency, estimation, and capacity limits," IEEE Transactions on Information Theory, vol. 60, pp. 7112-7139, Nov 2014.

[2] D. Gesbert, M. Shafi, D. shan Shiu, P. J. Smith, and A. Naguib, "From theory to practice: an overview of mimo space-time coded wireless systems," IEEE Journal on Selected Areas in Communications, vol. 21, pp. 281-302, Apr 2003.

[3] F. Rusek, D. Persson, B. K. Lau, E. G. Larsson, T. L. Marzetta, O. Edfors, and F. Tufvesson, "Scaling up mimo: Opportunities and challenges with very large arrays," IEEE Signal Processing Magazine, vol. 30, pp. 40-60, Jan 2013.

[4] V. Raghavan and A. M. Sayeed, "Sublinear capacity scaling laws for sparse mimo channels," IEEE Transactions on Information Theory, vol. 57, pp. 345-364, Jan 2011.

[5] L. Lu, G. Y. Li, A. L. Swindlehurst, A. Ashikhmin, and R. Zhang, "An overview of massive mimo: Benefits and challenges," IEEE Journal of Selected Topics in Signal Processing, vol. 8, pp. 742-758, Oct 2014.
[6] Y. Long, Z. Chen, and J. Fang, "Nonasymptotic analysis of capacit in massive mimo systems," IEEE Wireless Communications Letters, vol. 4, pp. 541-544, Oct 2015.

[7] P. Fernandes, J. Guerreiro, R. Dinis, and P. Montezuma, "On the capacity of nonlinear massive mimo-ofdm systems," in 2016 IEEE 84th Vehicular Technology Conference (VTC-Fall), pp. 1-5, Sept 2016.

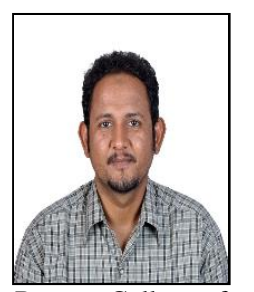

Mohammed Hyder Mekki Sadig has obtained his B.Sc. degree in Electrical and Electronic Engineering from Bayan College for Science \& Technology in 2009.

He has obtained his MSc. degree from Alzaiem Alazhari Univesity Electrical and Electronic Engineering department in 2014. He has been a PhD student Since then 2105 .

Mohamed worked and still working as lecturer in Bayan College for Science \& Technology, Alslama College, and Cambridge International College (Sudan) and supervised many final year graduation projects.

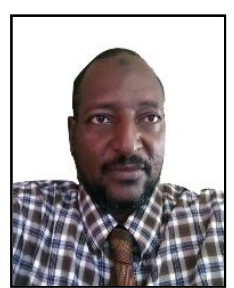

Associate Prof. Dr. Amin Babiker A/Nabi Mustafa has obtained his B.Sc. degree in Electrical and Electronic Engineering from Faculty of Engineering University of Khartoum in 1990.

$\mathrm{He}$ has obtained his Ph.D. degree from Alneelain University Faculty of Engineering in 2007. He has been the Dean, Faculty of Engineering Alneelain University since 2009 up to 2015 when he has taken the post of Secretary of Academic Affairs.

Prof Amin Supervised or Supervising more than 150 M.Sc. or Ph.D. Students. Dr. Amin has been appointed as External Examiner for so many Universities. He is a member of Sudan Engineering Council, Federation of Sudanese Engineers, Federation of Arab Engineers and its Engineering Education Committee.

Dr. Amin Published over 300 articles in International, Regional and Local Scientific Journals. He participated in several related international conferences. He has been the Co-chair of IEEE Conference organized by Alneelain University in 2015. 